ARTigo ORIGinal

Original Article

\title{
Perfil Gestacional na Espondilite Anquilosante $e^{(*)}$
}

\section{Presnancy Profile in Ankylosing Spondylitis}

\author{
Percival Degrava Sampaio-Barros ${ }^{(1)}$, Manoel Barros Bértolo ${ }^{(2)}$, Adil Muhib Samara ${ }^{(3)}$
}

\section{RESUMO}

Objetivo: avaliar o perfil gestacional numa casuística de mulheres com espondilite anquilosante (EA) acompanhadas em um hospital universitário de referência. Métodos: estudo retrospectivo avaliando 40 mulheres com EA, segundo os critérios modificados de New York, acompanhadas no Ambulatório de Espondiloartropatias da FCM-UNICAMP no período entre 1990 e 2004. Foram avaliados os dados referentes ao número de gestações, partos e abortos, bem como o comportamento da doença durante a gestação naquelas pacientes que engravidaram após o início da EA. Resultados: dentre as 40 mulheres avaliadas, 35 (87,5\%) eram brancas e 27 (67,5\%) eram HLA-B27 positivo, sendo que somente três $(7,5 \%)$ referiam história familiar de EA. A média de idade de início foi de 29,9 anos e o tempo médio de doença foi de 15,5 anos. Nove pacientes $(22,5 \%)$ nunca haviam engravidado, sendo que apenas uma paciente decidiu não engravidar por causa da EA. Trinta e uma pacientes apresentaram 83 gestações (média de 2,7 gestações/paciente), sendo sete após o início da doença. Ocorreram 71 partos e 12 abortos espontâneos (nenhum após o início da doença). Dentre as cinco pacientes que engravidaram após o início da EA, apenas uma apresentou piora da doença, associada ao comprometimento das coxofemorais. Conclusões: a análise do perfil gestacional da presente casuística indica que a fertilidade parece não ter sido afetada antes do início da EA e que a maioria das pacientes decide não engravidar após o início da doença.

Palavras-chave: espondilite anquilosante, sexo feminino, gestação, aborto.

\section{INTRODUÇÃO}

A espondilite anquilosante (EA) é uma doença inflamatória crônica que acomete preferencialmente a coluna vertebral.

\begin{abstract}
Objective: To analyze the pregnancy profile in a group of female patients with ankylosing spondylitis (AS) followed at a referral university center. Methods: Retrospective study analyzing 40 women with the diagnosis of AS according to the New York modified criteria followed at the Spondyloarthropathies Outpatient Clinic of the State University of Campinas in the period between 1990-2004. The number of pregnancies, deliveries and miscarriages was investigated, as well as the disease behavior during the pregnancy in those patients that got pregnant after AS onset. Results: Among the 40 AS patients, 35 (87.5\%) were Caucasians and 27 (67.5\%) were HLA-B27 positive; 3 patients (7.5\%) referred familial history of AS. The mean age of onset was 29.9 years old and the mean disease duration was 15.5 years. Nine patients (22.5\%) never got pregnant; just one patient decided not to get pregnant because of AS. Thirty-one patients presented 83 pregnancies (an average of 2.7 pregnancies/patient), being seven after disease onset. There were 70 deliveries and 12 spontaneous miscarriages, but no miscarriages after AS onset. Among the 5 patients that got pregnant after disease onset, just 1 patient referred worsening of AS, related to hip involvement. Conclusions: In the present casuistic, fertility seemed not to be affected before disease onset and most patients decided not to get pregnant after AS onset.
\end{abstract}

Keywords: ankylosing spondylitis, female sex, pregnancy, miscarriage.

Dentre os fatores epidemiológicos, a doença predomina no sexo masculino, em populações brancas, em adultos (entre 16 e 45 anos) e em populações onde ocorre maior prevalência do HLA-B27 $7^{(1)}$.

\footnotetext{
* Trabalho realizado na Disciplina de Reumatologia do Departamento de Clínica Médica da Faculdade de Ciências Médicas da Universidade Estadual de Campinas (FCM/UNICAMP), Campinas, SP, Brasil. Este trabalho contou com o apoio do Fundo de Auxílio à Pesquisa e Ensino em Reumatologia da Sociedade Brasileira de Reumatologia. Recebido em 04/1 1/2004. Aprovado, após revisão, em 18/02/2005.

1. Assistente-Doutor. FCM/UNICAMP.

2. Professor Assistente-Doutor. Chefe do Departamento de Clínica Médica. FCM/UNICAMP.

3. Professor Titular e Chefe da Disciplina de Reumatologia. FCM/UNICAMP.

Endereço para correspondência: Dr. Percival Degrava Sampaio-Barros. Disciplina de Reumatologia, Departamento de Clínica Médica. Faculdade de Ciências Médicas Universidade Estadual de Campinas (FCM/UNICAMP). Barão Geraldo, Campinas, SP, Brasil. CEP 13081-970. E-mail: psbarros@fcm.unicamp.br
} 
$\mathrm{Na}$ artrite reumatóide (AR), a gestação parece não afetar a progressão clínica e radiológica da doença ${ }^{(2)}$. Já na EA, que costuma se iniciar no adulto jovem, numa fase da vida em que a mulher costuma engravidar, os poucos estudos iniciais mostraram que a doença melhorava em 20\% a 25\% das pacientes, enquanto na grande maioria dos casos a EA permanecia inalterada ou piorava ${ }^{(3)}$. Em uma comparação das influências da gestação na evolução de pacientes com EA, AR juvenil e artrite psoriásica, foi demonstrada a ocorrência de surtos de atividade da doença nos três meses após o parto em $90 \%$ das pacientes espondilíticas, $70 \%$ das pacientes psoriásicas e $50 \%$ das pacientes com AR juvenil ${ }^{(4)}$.

Existem poucos estudos avaliando o perfil gestacional da mulher espondilítica em diferentes populações. Em 1998, foi publicada uma interessante pesquisa realizada em 13 países da América do Norte e Europa, junto às associações nacionais de pacientes com EA, com questionários respondidos por 939 pacientes. Foi observada a média de 2,4 gestações/paciente, num conjunto de 649 mulheres, ocorrendo 15,1\% de abortos; nas gestações ocorridas após o início da EA, 32,5\% referiram piora enquanto 33,2\% referiram que a doença permaneceu inalterada; 93,2\% dos partos foram a termo, com $58 \%$ de cesarianas $^{(5)}$.

Os autores apresentam a análise do perfil gestacional de uma casuística de 40 pacientes espondilíticas acompanhadas em um centro universitário de referência.

\section{MATERIAL E MÉTODOS}

No período entre janeiro de 1990 e dezembro de 2004, foram cadastrados 230 pacientes com diagnóstico de EA, segundo os critérios modificados de New York ${ }^{(6)}$, atendidos no Ambulatório de Espondiloartropatias do Hospital de Clínicas da UNICAMP. Dentre estes pacientes, 40 (17\%) eram do sexo feminino.

Os autores avaliaram de maneira retrospectiva os dados referentes ao número de gestações, partos e abortos das pacientes espondilíticas. As pacientes que engravidaram após o início da EA foram questionadas sobre o comportamento da doença durante a gestação. As pacientes também foram inquiridas com relação à história familiar de EA.

\section{RESULTADOS}

Dentre as 40 mulheres avaliadas, $35(87,5 \%)$ eram brancas e $27(67,5 \%)$ eram HLA-B27 positivo, sendo que somente três $(7,5 \%)$ referiam história familiar de EA em parente consangüíneo de primeiro grau (pai e/ou irmão). A média de idade foi de 45,4 anos (variando entre 21 e 71 anos) e o tempo médio de doença foi de 15,5 anos; a média de idade de início foi de 29,9 anos. Acometimento axial isolado foi observado em 15 pacientes (37,5\%). Três pacientes $(7,5 \%)$ apresentavam a característica "coluna em bambu". Acometimento de articulações de cinturas e periféricas foram observadas em 25 pacientes $(62,5 \%)$, destacando-se o envolvimento de tornozelos $(47,5 \%)$, entesopatia aquileana e/ou plantar (35\%), joelhos (25\%), coxofemorais (15\%, com prótese bilateral de quadris em três pacientes), esternoclaviculares $(15 \%)$ e ombros (5\%).

Nove pacientes $(22,5 \%)$ nunca engravidaram: quatro pacientes com doença leve e menos de 30 anos de idade ainda se consideram jovens para a maternidade; duas pacientes casadas com mais de 35 anos não puderam ter filhos, e não sabem o motivo da infertilidade; duas pacientes referiram que pretendem continuar solteiras e não ter filhos, independente da doença; uma paciente nulípara com quadro axial e periférico intensos decidiu não engravidar por causa da EA. Dentre as restantes 31 pacientes, que tiveram no mínimo uma gestação, 13 iniciaram a doença após os 35 anos, quando já não mais planejavam ter filhos.

Trinta e uma pacientes apresentaram 83 gestações (média de 2,7 gestações/paciente), sendo sete após o início da doença. Ocorreram 71 partos $(85,5 \%)$, nenhum gemelar. Houve 12 abortos (14,5\%), todos espontâneos, nenhum após o início da doença. Não foi relatado nenhum caso de natimorto ou de malformação congênita.

Dentre as cinco pacientes que engravidaram após o início da EA, apenas uma apresentou piora da doença, associada ao comprometimento das coxofemorais, tendo de permanecer o último trimestre gestacional em repouso absoluto, mas sem prejuízo para o feto, que nasceu com peso e estatura normais, de parto cesariano, sendo posteriormente encaminhada para prótese bilateral de quadris. Nas outras quatro pacientes, com doença predominantemente axial e sintomas de leve intensidade, a doença tendeu a permanecer inalterada, sendo que duas pacientes engravidaram duas vezes; nenhuma das cinco pacientes planejava engravidar novamente.

\section{DISCUSSÃO}

Existem divergências quanto à apresentação e evolução da EA no sexo feminino. Estudos europeus revelam que a razão por sexo (masculino:feminino) vem progressivamente diminuindo nas últimas décadas ${ }^{(7,8)}$, e que a doença na mulher tende a ser semelhante à do homem ${ }^{(9-11)}$. Já nas 
casuísticas de pacientes das Américas, mesmo em populações distintas como os mestiços mexicanos ${ }^{(12)}$, os esquimós do Alasca $^{(13)}$ e as do sudeste do Brasil ${ }^{(14)}$, a EA tende a ser mais branda no sexo feminino. Em trabalho recente, avaliando os padrões da doença em um grupo de 147 pacientes espondilíticos acompanhados na Universidade Estadual de Campinas, foi observado que o sexo feminino estava estatisticamente associado ao acometimento das articulações esternoclaviculares, apresentando menor freqüência do acometimento de coluna dorsal e cervical e quadris ${ }^{(14)}$. No presente estudo, a razão por sexo da casuística avaliada foi de 4,75 homens:1 mulher, semelhante à observada no mesmo serviço nas últimas duas décadas. Apenas três pacientes (7,5\%) apresentaram a característica "coluna-em-bambu", um valor bem inferior àquele observado quando se acrescenta os pacientes do sexo masculino avaliados no mesmo serviço (20\%).

Calin et al. ${ }^{(15)}$, analisando um banco de dados de 4.400 pacientes na Inglaterra, postularam que a influência do sexo feminino é maior que a do sexo masculino na determinação da suscetibilidade à EA nos filhos, baseados na elevada prevalência de pacientes do sexo feminino em casos com história familiar de EA; posteriormente, este estudo foi estendido para as demais espondiloartropatias ${ }^{(16)}$. JiménezBalderas et al. ${ }^{(17)}$, estudando a família de 40 pacientes espondilíticos com história familiar de EA, observaram associação estatística entre EA de início juvenil e história familiar do lado materno, em mães mais jovens. Na presente casuística, história familiar de EA foi referida por apenas três pacientes $(7,5 \%)$, que apresentavam familiares de primeiro grau do sexo masculino (pai ou irmão) com a doença. Como muitas das pacientes apresentam filhos e filhas ainda bastante jovens, a maioria com menos de 25 anos de idade, a prevalência de história familiar positiva pode aumentar após um seguimento mais longo. A hipótese de que o primeiro filho apresenta maior risco de desenvolver a EA que os outros filhos, como observado em modelos experimen-

\section{REFERÊNCIAS}

1. Olivieri I, Van Tubergen A, Salvarani C, Van der Linden S: Seronegative spondyloarthritides. Best Pract Res Clin Rheumatol 16: 723-39, 2002.

2. Drossaers-Bakker KW, Zwinderman AH, Van Zeben D, Breedveld FC, Hazes JMW: Pregnancy and oral contraceptive use do not significantly influence outcome in long term rheumatoid arthritis. Ann Rheum Dis 61: 405-8, 2002.

3. Ostensen M, Husby G: Ankylosing spondylitis and pregnancy Rheum Dis Clin North Am 15: 241-54, 1989. tais em camundongos, ainda é bastante controversa na literatura ${ }^{(18,19)}$.

Infertilidade não foi evidenciada objetivamente em nenhuma das 40 pacientes espondilíticas analisadas neste estudo, e não costuma ser referida na literatura ${ }^{(5)}$. A taxa de fertilidade foi de 2,7 gestações/paciente, semelhante à taxa observada no Brasil em 2003, que é de 2,1 gestações/mulher, segundo dados recentes do Instituto Brasileiro de Geografia e Estatística $(\mathrm{IBGE})^{(20)}$. A taxa de abortos observada antes do início da EA $(14,5 \%)$ também é semelhante à observada no país.

Após o início da doença, somente cinco pacientes engravidaram, com a gestação transcorrendo bem em todas as pacientes, não ocorrendo nenhum aborto; uma única paciente teve piora significativa da doença durante a gestação, associada à piora do acometimento de coxofemorais. O grande número de recidivas pós-parto observado no estudo de $\varnothing_{\text {stensen }}{ }^{(5)}$ não foi verificado neste estudo. Recente estudo demonstrou que ocorre aumento das citocinas próinflamatórias durante a gestação de pacientes com EA e $\mathrm{AR}$, e que estas tendem a diminuir após o parto ${ }^{(21)}$. Outra importante consideração no manuseio das pacientes espondilíticas é representada pelos cuidados anestésicos ${ }^{(22)}$. No trabalho de $\varnothing_{\text {stensen }}{ }^{(5)}$, a taxa de cesarianas (58\%) foi consideravelmente maior na população de mulheres espondilíticas que aquela observada na população dos países europeus onde a pesquisa foi realizada.

O número de gestações após o início da EA no presente estudo é muito pequeno para se tirar conclusões definitivas sobre o comportamento da EA durante a gestação. No entanto, a análise crítica da literatura não contra-indica a gestação na EA, mas deve-se ter cautela quanto à liberação da gestação em pacientes com acometimento importante das articulações periféricas e das cinturas, notadamente coxofemorais. Como a EA ocorre em pacientes jovens, a mulher espondilítica deve ser cuidadosamente informada sobre a evolução da doença e os problemas que eventualmente poderão ocorrer durante sua gestação, quando o esquema terapêutico deve ser adaptado.

4. Østensen M: The effect of pregnancy on ankylosing spondylitis, psoriatic arthritis, and juvenile rheumatoid arthritis. Am J Reprod Immunol 28: 235-7, 1992.

5. Østensen M, Østensen H: Ankylosing spondylitis - the female aspect. J Rheumatol 25: 120-4, 1998.

6. Van der Linden S, Valkenburg HA, Cats A: Evaluation of diagnostic criteria for ankylosing spondylitis: A proposal for modification of the New York criteria. Arthritis Rheum 27: 361-8, 1984.

7. Will R, Edmunds L, Elswood J, Calin A: Is there sexual inequality in ankylosing spondylitis? A study of 498 women and 1202 men. J Rheumatol 17: 1649-52, 1990. 
8. Kennedy LG, Will R, Calin A: Sex ratio in the spondyloarthropathies and its relationship to phenotypic expression, mode of inheritance and age at onset. J Rheumatol 20: 1900-4, 1993.

9. Gran JT, ØstensenM, Husby G: A clinical comparison between males and females with ankylosing spondylitis. J Rheumatol 12: 126-9, 1985.

10. Kidd B, Mullee M, Frank A, Cawley M: Disease expression of ankylosing spondylitis in males and females. J Rheumatol 15: 1407-9, 1988.

11. Eustace S, Coughlan RJ, McCarthy C: Ankylosing spondylitis: A comparison of clinical and radiographic features in men and women. Ir Med J 86: 120-2, 1993.

12. Jiménez-Balderas FJ, Mintz G: Ankylosing spondylitis: clinical course in women and men. J Rheumatol 20: 2069-72, 1993.

13. Boyer GS, Templin DW, Bowler A, et al: Spondyloarthropathy in the community: Differences in severity and disease expression in Alaskan Eskimo men and women. J Rheumatol 27: 170-6, 2000.

14. Sampaio-Barros PD, Bértolo MB, Kraemer MHS, Marques Neto JF, Samara AM: Primary ankylosing spondylitis: Patterns of disease in a Brazilian population of 147 patients. J Rheumatol 28: 560-5, 2001.

15. Calin A, Brophy S, Blake D: Impact of sex on inheritance of ankylosing spondylitis: a cohort study. Lancet 354: 1687-90, 1999.
16. Brophy S, Taylor G, Blake D, Calin A: The interrelationship between sex, susceptibility factors, and outcome in ankylosing spondylitis and its associated disorders including inflammatory bowel disease, psoriasis, and iritis. J Rheumatol 30: 2054-8, 2003.

17. Jiménez-Balderas FJ, Zonana-Nacach A, Sánchez ML, et al: Maternal age and family history are risk factors for ankylosing spondylitis. J Rheumatol 30: 2182-5, 2003.

18. Baudoin P, Van der Horst-Bruinsma IE, Dekker-Saeys AJ, Weinreich S, Bezemer PD, Dijkmans BAC: Increased risk of developing ankylosing spondylitis among first-born children. Arthritis Rheum 43: 2818-22, 2000.

19. Brophy S, Taylor G, Calin A: Birth order and ankylosing spondylitis: No increased risk of developing ankylosing spondylitis among firstborn children. J Rheumatol 29: 527-9, 2002.

20. Indicadores Demográficos IBGE: Taxa de fecundidade total 2003. www.ibge.gov.br/brasil_em_sintese.

21. Østensen M, Foerger F, Nelson JL, Schumacher A, Hebisch G, Villiger PM: Pregnancy in patients with rheumatic disease: antiinflammatory cytokines increase in pregnancy and decrease post partum. Ann Rheum Dis 64: 839-44, 2005.

22. Bourlier RA, Birnbach DJ: Anesthetic management of the parturient with ankylosing spondylitis. Int J Obstet Anesth 4: 244-7, 1995. 